\title{
Evaluation of Five Ultrasound Risk-Stratification Systems for Choosing Thyroid Nodules for Fine- Needle Aspiration
}

\section{Stephanie A. Fish}

Department of Endocrinology, Memorial Sloan Kettering Cancer Center, New York, New York, U.S.A.

Review of: Castellana M, Castellana C, Treglia G, Giorgino F, Giovanella L, Russ G, Trimboli P 2019 Performance of five ultrasound risk stratification systems in selecting thyroid nodules for FNA: a metaanalysis. J Clin Endocrinol Metab. Epub 2019 Nov 6. PMID: 31690937.

\section{SUMMARY}

\section{Background}

Thyroid nodules are common. While most nodules are benign, approximately 7 to $10 \%$ of them are malignant (1). The goal in the evaluation of thyroid nodules is to identify the suspicious nodules that require further testing from the benign nodules that can be monitored. Ultrasound is the imaging method recommended for the evaluation of thyroid nodules. Specific sonographic features associated with malignancy include hypoechogenicity, taller-than wide shape, irregular margins, microcalcifications, and extrathyroidal extension of the thyroid nodule (2). Several ultrasound risk-stratification systems (RSSs) have been developed that use nodule appearance to determine the risk of malignancy and to make suggestions regarding the need for fine-needle aspiration (FNA). Two of these systems are part of clinical guidelines (3, and three are part of radiological recommendations (5-7). Previous retrospective studies conducted to determine the best ultrasound system to evaluate thyroid nodules have been heterogeneous and limited by selection bias.

This study is a meta-analysis to evaluate the diagnostic performance of the five ultrasound RSSs (8). The primary outcomes were the diagnostic odds ratio (DOR) of each system taken independently and the relative diagnostic odds ratio (RDOR) when head- to-head comparison was feasible. The secondary outcomes were to evaluate sensitivity, specificity, positive predictive value (PPV), negative predictive value (NPV), and likelihood ratio for positive (LR+) and for negative (LR-) results.

\section{Methods}

For this meta-analysis, a comprehensive search strategy was completed in PubMed. Studies reporting the diagnostic performance of at least one of the ultrasound RSSs in thyroid nodules were included if they met the following criteria: (1) the diagnosis of benign nodules was based on histology, core-needle biopsy, or cytology and; (2) the diagnosis of malignant nodules was not based on cytology only. Studies were excluded if they focused on pediatric patients or included only cytologically indeterminate thyroid nodules. Studies were also excluded if they used cytology alone for determining both benign and malignancy nodules.

Data from each study was extracted independently by two investigators. A benign nodule was classified as true negative if FNA was not indicated by the specific ultrasound RSS and as false positive if FNA was indicated by the specific ultrasound RSS. A malignant nodule was classified as true positive if FNA was indicated by the specific ultrasound RSS 

Stratification Systems for Choosing Thyroid Nodules for Fine-Needle Aspiration

and as false negative if FNA was not indicated by the specific ultrasound RSS. A diagnostic performance meta-analysis in selecting nodules for FNA was carried out. Then, a head-to-head comparison on the accuracy of ultrasound RSSs was performed if at least five studies were available.

\section{Results}

A total of 854 papers were identified in the initial search. After the removal of duplicate studies and the exclusion of ineligible studies, 79 papers were reviewed in full. After consideration of study criteria, 12 studies from this pool were included in the final analysis. The papers, which were published between 2017 and 2019, had sample sizes ranging from 424 to 4696 thyroid nodules. Participants were adult outpatients who underwent either thyroid nodule FNA, core needle biopsy, or thyroid surgery and had ultrasound images available. Thyroid nodules diagnosed as indeterminate on FNA were generally excluded from the study, unless a final surgical pathologic diagnosis was available. Nine studies were retrospective and two prospective cohorts. One study did not clearly state its design. Three studies each were completed in China, South Korea, and Italy and one each in the United States, Canada, France, Switzerland, and the United Kingdom. Four studies assessed the ultrasound RSSs from the American Association of Clinical Endocrinologists, American College of Endocrinology, and Associazione Medici Endocrinologi; seven using ACR-TIRADS; nine from the American Thyroid Association; three using EU-TIRADS, and five using K-TIRADS (some studies used more than one of these systems). The prevalence of malignancy ranged from $4 \%$ to $54 \%$. Overall, 4378 malignant and 14,372 benign nodules were included in the review.

The pooled sensitivities ranged from $54 \%$ to $87 \%$, specificities from $28 \%$ to $64 \%$, PPVs from $17 \%$ to $43 \%$, and NPVs from $81 \%$ to $93 \%$. The pooled LR+ ranged from 1.2 to 1.9 , LR- from 0.4 to 0.6 , and DOR from 2.2 to 4.9. There was a high amount of heterogeneity for all the outcomes.

The investigators were able to do a comparison on the accuracy of the ultrasound RSSs when data from at least five studies were available (ATA, ACR-TIRADS, and K-TIRADS). When they considered only studies assessing simultaneously all evaluated ultrasound RSSs through a head-to-head comparison, a higher RDOR was found for ACR-TIRADS than for ATA $(P=0.002)$ or K-TIRADS $(P=0.002)$. A higher relative $L R+$ was found for ACR-TIRADS than for ATA $(P<0.001)$ or K-TIRADS $(P<0.001)$, as well as for ATA versus $K$-TIRADS $(P=0.048)$. No difference in relative $L R-$ was found.

\section{Conclusions}

This meta-analysis found a higher performance of ACR-TIRADS in selecting thyroid nodules for FNA. However, the comparison across the most commonly used ultrasound RSSs was limited owing to the limited data available.

\section{COMMENTARY}

This meta-analysis was designed to evaluate the effectiveness of the five most common ultrasound RSSs used in identifying thyroid nodules for FNA. After a comprehensive literature search, the authors included 12 studies assessing 4378 malignant and 14,372 benign nodules. Overall, there were more data on the use of ACR-TIRADS, ATA, and K-TIRADS. There were limited data on AACE/ACE/ AME and EU-TIRADS. The analysis showed a high variability in the performance of the five ultrasound 

Stratification Systems for Choosing Thyroid Nodules for Fine-Needle Aspiration

RSSs. However, the NPV was high in almost every system. The results of LR+ and LR- showed weak evidence across all ultrasound RSSs for the effectiveness to correctly select thyroid nodules for FNA. The authors did find that ACR-TIRADS performed better than ATA or K-TIRADS. This is likely due to a better ability of ACR-TIRADS to select malignant nodules for FNA. No difference was found between the ultrasound RSSs in identifying benign nodules.

This is a clinically important study, as thyroid nodules are a common clinical finding. To appropriately evaluate thyroid nodules, it is important to identify the nodules most at risk for thyroid cancer. Ultrasound is the imaging method of choice to evaluate thyroid nodules. The challenge for clinicians is that there are five different published systems for using ultrasound in determining which nodules should be evaluated with FNA. In this study, the authors try to identify the most accurate ultrasound RSS for identifying thyroid nodules for FNA. Overall, the authors found few studies that were appropriate to include in the study; in particular, there were very few data on the AACE/ACE/AME and EU-TIRADS systems. With this limited data, they found that the ACR-TIRADs performed the best. But, the strength of this result is limited owing to the significant limitations to the study. Most of the studies included in the analysis were retrospective in design, which can lead to selection bias. In addition, in some included studies, the ultrasound images were retrospectively reviewed to provide appropriate categorization, which can lead to additional bias in the results. Most importantly, there were only 12 studies included in the analysis and some of the ultrasound RSSs had very limited data. Given these limitations, it is difficult to draw clear conclusions.

A risk-stratified approach to the evaluation of thyroid nodules is appropriate and efficient. However, at this time, it is not clear which ultrasound RSS is most effective in performing this risk stratification. This study does show that ACR-TIRADs appears to be the most accurate system. However, additional prospective studies are needed to confirm these findings. Of note, the five ultrasound RSSs are quite similar. A collaborative effort to establish a single system for ultrasound evaluation of thyroid nodules would be the most effective and efficient for both the patient and the clinician. The hope is that this will be developed in the future.

\section{References}

1. Durante C, Grani G, Lamartina L, Filetti S, Mandel SJ, Cooper DS 2018 The diagnosis and management of thyroid nodules: a review. JAMA 319:914-924.

2. Remonti LR, Kramer CK, Leitao CB, Pinto LC, Gross JL 2015 Thyroid ultrasound features and risk of carcinoma: a systematic review and meta-analysis of observational studies. Thyroid 25:538-550.
3. Gharib H, Papini E, Garber JR, Duick DS, Harrell RM, Hegedüs L, Paschke R, Valcavi R, Vitti P; AACE/ACE/ AME Task Force on Thyroid Nodules. 2016 American Association of Clinical Endocrinologists, American College of Endocrinology, and Associazione Medici Endocrinologi Medical Guidelines for clinical practice for the diagnosis and management of thyroid nodules-2016 update. Endocr Pract 22:622-639. 
THYROID NODULES Evaluation of Five Ultrasound RiskStephanie A. Fish Stratification Systems for Choosing Thyroid Nodules for Fine-Needle Aspiration

4. Haugen BR, Alexander EK, Bible KC, Doherty GM, Mandel SJ, Nikiforov YE, Pacini F, Randolph GW, Sawka AM, Schlumberger M, et al. 2016 2015 American Thyroid Association management guidelines for adult patients with thyroid nodules and differentiated thyroid cancer: the American Thyroid Association Guidelines Task Force on Thyroid Nodules and Differentiated Thyroid Cancer. Thyroid 26:1-133.

5. Shin JH, Baek JH, Chung J, Ha EJ, Kim JH, Lee YH, Lim HK, Moon WJ, Na DG, Park JS, et al. 2016 Ultrasonography diagnosis and imaging-based management of thyroid nodules: revised Korean Society of thyroid radiology consensus statement and recommendations. Korean J Radiol 17:370-395.

6. Russ G, Bonnema SJ, Erdogan MF, Durante C, Ngu R, Leenhardt L 2017 European Thyroid Association Guidelines for Ultrasound Malignancy Risk Stratification of Thyroid Nodules in Adults: The EUTIRADS. Eur Thyroid J 6:225-237.
7. Tessler FN, Middleton WD, Grant EG, Hoang JK, Berland LL, Teefey SA, Cronan JJ, Beland MD, Desser TS, Frates MC, et al. 2017 ACR Thyroid Imaging, Reporting and Data System (TI-RADS): white paper of the ACR TI-RADS Committee. J Am Coll Radiol 14:587-595.

8. Castellana M, Castellana C, Treglia G, Giorgino F, Giovanella L, Russ G, Trimboli P 2019 Performance of five ultrasound risk stratification systems in selecting thyroid nodules for FNA: a meta-analysis. J Clin Endocrinol Metab. Epub 2019 Nov 6.

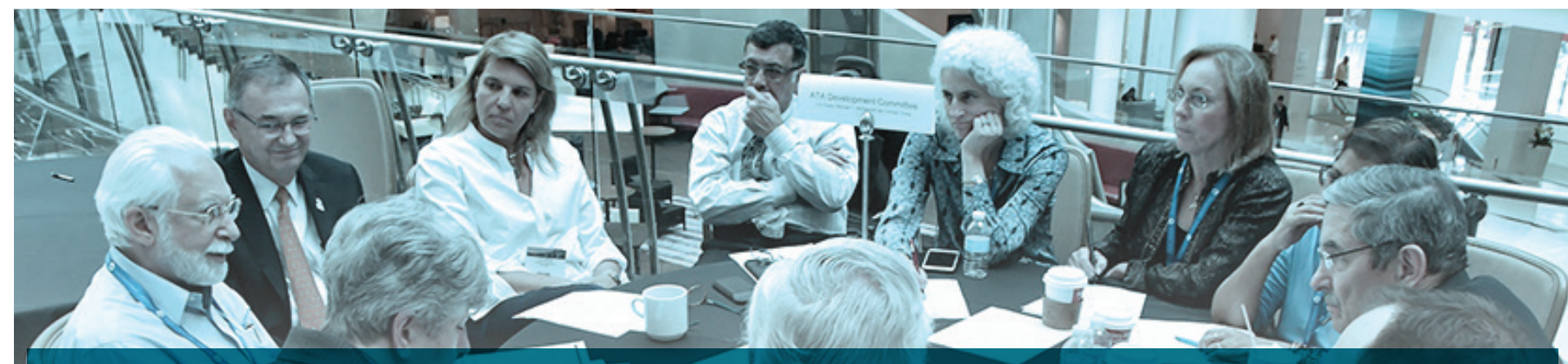

\section{Controversies in Thyroidology}

Spring Meeting of the American Thyroid Association ${ }^{\circledR}$ May 28-30, 2020 Westin New York Times Square 